\title{
ATTRACTORS OF ITERATED FUNCTION SYSTEMS
}

\author{
P. F. DUVALL, JR. AND L. S. HUSCH
}

(Communicated by James E. West)

\begin{abstract}
In this paper, the question of which compact metric spaces can be attractors of hyperbolic iterated function systems on Euclidean space is studied. It is shown that given any finite-dimensional compact metric $X$, there is a Cantor set $C$ such that the disjoint union of $C$ and $X$ is an attractor. In the process, it is proved that every such $X$ is the Lipschitz image of a Cantor set.
\end{abstract}

\section{INTRODUCTION}

If $X$ is a complete metric space and $F=\left\{f_{1}, f_{2}, \ldots, f_{k}\right\}$ is a collection of contraction mappings of $X$ to itself, then $F$ is said to be a (hyperbolic) Iterated Function System. It is well known [4] that for such an $F$ there is a unique compact set $A$ such that $A=\bigcup_{i=1}^{k} f_{i}(A)$. $A$ is called the attractor for $F$. In this paper, we are concerned with the question of which compact sets in Euclidean space can be realized as the attractors of some IFS. Williams [6] initially investigated the topological structure of attractors of IFS's. Hata [3] generalized Williams' work and showed that not every compactum can be realized as the attractor of an IFS, since, for example, a connected attractor must be locally connected. Hata posed the question whether every finite-dimensional locally connected continuum is the attractor of some IFS. Barnsley [1] showed to what extent every compactum can be approximated by an attractor of an IFS. As a special case, we note that it is not difficult to show that every compact subpolyhedron in $\mathbf{R}^{n}$ is an attractor.

We show (Theorem 4.1) that for each $n>0$ there is a Cantor set $C^{n} \subset \mathbf{R}^{2 n}$ with the property that if $X$ is any compact set in $\mathbf{R}^{n}$, the set

$$
C^{n} \times\{0\} \cup\{0\} \times X \subset \mathbf{R}^{3 n}
$$

is the attractor of an IFS. It follows that every compact, finite dimensional metric space that contains a closed and open Cantor subset can be embedded in some Euclidean space as an attractor.

Received by the editors February 11, 1991.

1991 Mathematics Subject Classification. Primary 54H15; Secondary 54H20, 54E40.

Key words and phrases. Iterated function systems, attractors, fractal geometry, contractions, Lipschitz mappings.

This research was done while L. Husch was a Distinguished Visiting Professor at the University of North Carolina, Greensboro; support is gratefully acknowledged. 
Most of our notation is standard, although we shall use $\|\cdot\|$ to denote the sum norm on $\mathbf{R}^{n}$. That is, if $x=\left(x_{1}, x_{2}, \ldots, x_{n}\right)$ then $\|x\|=\left|x_{1}\right|+\left|x_{2}\right|+$ $\cdots+\left|x_{n}\right|$.

\section{LIPSCHITZ MAPPINGS OF CANTOR SETS ONTO COMPACTA}

A classical theorem of topology states that every compact metric space is the continuous image of the Cantor set. In this section, we construct, for each $n>0$, a Cantor set $V^{n}$ with the property that for every compactum $X \subset \mathbf{R}^{n}$ there is a Lipschitz mapping of $V^{n}$ onto $X$.

To begin, fix an $n \geq 1$ and let $B$ be the unit cube in $\mathbf{R}^{n}$ spanned by the standard basis vectors. Subdivide $B$ into cubes $B_{i}, i \in \Gamma$, where $\Gamma=$ $\left\{0,1, \ldots, 2^{n}-1\right\}$ and $B_{i}=\prod_{j=0}^{n}\left[\varepsilon_{j}, 1 / 2+\varepsilon_{j}\right]$ with $\varepsilon_{j}$ being one half the $j$ th binary digit of $i$. Subdivide the $B_{i}$ 's in a similar manner and continue the process so that at the $k$ th stage, $B$ is the union of $2^{n k}$ cubes $B_{i_{1} i_{2} \cdots i_{k}}$. For each $k \geq 1$, give the space of $k$-tuples of integers the discrete topology, let

$$
V_{k}=\bigcup_{i_{1}, i_{2}, \ldots, i_{k} \in \Gamma} B_{i_{1} i_{2} \cdots i_{k}} \times\left(i_{1}, i_{2}, \ldots, i_{k}\right)
$$

and define $p_{k}: V_{k+1} \rightarrow V_{k}$ by $p_{k}\left(x, i_{1}, i_{2}, \ldots, i_{k+1}\right)=\left(x, i_{1}, i_{2}, \ldots, i_{k}\right)$. If $\vec{i}=\left\{i_{k}\right\}$ is a sequence of integers with $i_{k} \in \Gamma$, let

$$
x(\vec{i})=\bigcap_{k=1}^{\infty} B_{i_{1} i_{2} \cdots i_{k}} .
$$

In the next section, we will need the following observation, whose proof is left to the reader.

Proposition 2.1. If $i_{k}=x_{k 1}+x_{k 2} 2+\cdots+x_{k n} 2^{n-1}$, with $x_{k i} \in\{0,1\}$, then the mth component of $x(\vec{i})$ has the base 2 expansion . $x_{1 m} x_{2 m} x_{3 m} \cdots$.

Let $V^{n}$ be the inverse limit of the sequence

$$
V_{1} \stackrel{p_{1}}{\leftarrow} V_{2} \stackrel{p_{2}}{\leftarrow} \cdots .
$$

Note that we may view a point in $V^{n}$ as a pair $(x, \vec{i})$, where $\vec{i}$ is a sequence of integers from $\Gamma$ and $x=x(\vec{i})$. Define a metric $\rho$ on $V^{n}$ by

$$
\rho((x, \vec{i}),(y, \vec{j}))=\|x-y\|+1 / 2^{k},
$$

where $\|\cdot\|$ is the sum norm described in the introduction and $k+1$ is the first index in which $\vec{i}$ and $\vec{j}$ disagree (note that if $\vec{i}=\vec{j}$ then $x=y$ ).

Proposition 2.2. $V^{n}$ is a Cantor set and $\rho$ is a metric consistent with the inverse limit topology on $V^{n}$. 
Proof. Recall that if $\left\{X_{k}\right\}$ is a sequence of metric spaces with bounded metrics $d_{k}$, then

$$
d\left(\left\{x_{k}\right\},\left\{y_{k}\right\}\right)=\sum_{k=1}^{\infty} \frac{d_{k}\left(x_{k}, y_{k}\right)}{2^{k}}
$$

is a metric on the product of the $X_{k}$ 's that induces the product topology. Let $d_{k}$ be the metric on $V_{k}$ given by

$$
d_{k}\left(\left(x, i_{1}, i_{2}, \ldots, i_{k}\right),\left(y, j_{1}, j_{2}, \ldots, j_{k}\right)\right)=\|x-y\|+\varepsilon
$$

where $\varepsilon=0$ if $\left(i_{1}, i_{2}, \ldots, i_{k}\right)=\left(j_{1}, j_{2}, \ldots, j_{k}\right), 1$ otherwise. Then, if $(x, \vec{i})$ and $(y, \vec{j})$ are in $V^{n}$ and $k+1$ is the first index for which $\vec{i}$ and $\vec{j}$ disagree, the construction of $d$ above gives

$$
\begin{aligned}
d((x, \vec{i}),(y, \vec{j})) & =\sum_{m=1}^{\infty} \frac{\|x-y\|}{2^{m}}+\sum_{m=k+1}^{\infty} \frac{1}{2^{m}} \\
& =\|x-y\|+\frac{1}{2^{k}}=\rho((x, \vec{i}),(y, \vec{j})) .
\end{aligned}
$$

Thus $\rho$ agrees with the product metric. To see that $V^{n}$ is a Cantor set, note that the map $(x, \vec{i}) \rightarrow \vec{i}$ is a homeomorphism of $V^{n}$ onto the space of sequences of integers from $\Gamma$.

Now let $X$ be a compact subset of $B$, and define a mapping $\phi: V^{n} \rightarrow X$ as follows. For each $k$-tuple $\left(i_{1}, i_{2}, \ldots, i_{k}\right)$ such that $B_{i_{1} i_{2} \ldots i_{k}}$ intersects $X$, choose a point $x_{i_{1} i_{2} \cdots i_{k}} \in B_{i_{1} i_{2} \cdots i_{k}} \cap X$. Given $(x, \vec{i}) \in V^{n}$, define $\phi(x, \vec{i})= \begin{cases}x_{i_{1} i_{2} \cdots i_{k}} & \text { if } x \notin X \text { and } k \text { is the last index with } X \cap B_{i_{1} i_{2} \cdots i_{k}} \neq \varnothing, \\ x & \text { if } x \in X .\end{cases}$

Theorem 2.1. $\phi$ is a Lipschitz map of $V^{n}$ onto $X$.

Proof. Given $a=(x, \vec{i}), b=(y, \vec{j}) \in V^{n}$, there are three cases to consider.

Case 1. Both $x$ and $y$ are in $X$. In this case,

$$
\|\phi(a)-\phi(b)\|=\|x-y\| \leq \rho(a, b) .
$$

Case 2. $x \in X$, but $y \notin X$. Let $k+1$ be the first index in which $\vec{i}$ and $\vec{j}$ disagree, and let $\phi(b)=x_{j_{1} j_{2} \cdots j_{l}}$. Then $k \leq l$ and

$$
\left\|y-x_{j_{1} j_{2} \cdots j_{l}}\right\| \leq n / 2^{l} \leq n / 2^{k}
$$

since both $y$ and $x_{j_{1} j_{2} \cdots j_{l}}$ are in $B_{j_{1} j_{2} \cdots j_{l}}$. We have

$$
\begin{aligned}
\|\phi(a)-\phi(b)\| & =\left\|x-x_{j_{1} j_{2} \cdots j_{l}}\right\| \leq\|x-y\|+\left\|y-x_{j_{1} j_{2} \cdots j_{l}}\right\| \\
& \leq\|x-y\|+n / 2^{k} \leq n \rho(a, b) .
\end{aligned}
$$

Case 3. Neither $x$ nor $y$ are in $X$. Let $\phi(a)=x_{i_{1} i_{2} \cdots i_{l}}, \phi(b)=x_{j_{1} j_{2} \cdots j_{m}}$, and let $k+1$ be the first index in which $\vec{i}$ and $\vec{j}$ disagree. We may assume that $l \leq m$, and we would like to have $k \leq l$. If $m \leq k$, then $\phi(a)=\phi(b)$ 
and there is nothing to prove. By the definition of $\phi$, the case $l<k<m$ leads to a contradiction, so we assume $k \leq l$. Then

$$
\begin{aligned}
\|\phi(a)-\phi(b)\| & =\left\|x_{i_{1} i_{2} \cdots i_{l}}-x_{j_{1} j_{2} \cdots j_{m}}\right\| \\
& \leq\|x-y\|+\left\|x-x_{i_{1} i_{2} \cdots i_{l}}\right\|+\left\|y-x_{j_{1} j_{2} \cdots j_{m}}\right\| \\
& \leq\|x-y\|+2 n / 2^{k} \leq 2 n \rho(a, b) .
\end{aligned}
$$

Thus $\phi$ is a Lipschitz map with Lipschitz constant at most $2 n$.

Since every compact set in $\mathbf{R}^{n}$ can be moved into $B$ by similarity transformations, we have

Corollary 2.1. If $X$ is any compact set in $\mathbf{R}^{n}$, there is a Lipschitz map of $V^{n}$ onto $X$.

\section{Standard Cantor sets}

Let $C^{1}$ be the set of points in $\mathbf{R}^{2}$ of the form $(x, y)$, where $x, y \in[0,1]$ have base 2 expansions of the form $x=. x_{1} x_{2} x_{3} \cdots$ and $y=y_{1} y_{2} y_{3} \cdots$, with $x_{i}=0$ for $i$ even and $y_{i}=0$ for $i$ odd. Define contractions $h_{0}$ and $h_{1}$ on $\mathbf{R}^{2}$ by

$$
h_{0}(x, y)=\left(\frac{y}{2}, \frac{x}{2}\right) \text { and } h_{1}(x, y)=\left(\frac{y}{2}+\frac{1}{2}, \frac{x}{2}\right) .
$$

Proposition 3.1. $C^{1}$ is a Cantor set and is the attractor for the IFS $F^{1}=$ $\left\{h_{0}, h_{1}\right\}$.

Proof. It is easy to check that $h_{0}\left(C^{1}\right)$ and $h_{1}\left(C^{1}\right)$ are disjoint and that their union is $C^{1}$. Thus $C^{1}$ is the attractor and the disjointness implies that it is a Cantor set [3, Theorem 4.4].

If $F=\left\{f_{1}, f_{2}, \ldots, f_{k}\right\}$ and $G=\left\{g_{1}, g_{2}, \ldots, g_{l}\right\}$ are IFSs on the complete metric spaces $X$ and $Y$ with attractors $A$ and $B$, respectively, define the IFS $F \times G$ on $X \times Y$ to be the set of all products $f_{i} \times g_{j}$.

The reader can easily verify the following proposition.

Proposition 3.2. The attractor of $F \times G$ is $X \times Y$.

It follows that for each $n>1$ the $n$-fold product $C^{n}=C^{1} \times C^{1} \times \cdots \times C^{1}$ is a Cantor set in $\mathbf{R}^{2 n}$ that is the attractor of the IFS $F^{n}=F^{1} \times F^{1} \times \cdots \times F^{1}$.

We note in passing that since $F^{n}$ consists of $2^{n}$ similitudes with similarity constant $\frac{1}{2}$, the Hausdorff dimension of $C^{n}$ is $n$ [2].

In the previous section, we showed that each compactum in a finite-dimensional Euclidean space is the Lipschitz image of an abstractly defined Cantor set. The purpose of the present construction is to be able to replace $V^{n}$ by the more geometrically appealing $C^{n}$. Again, we need some preliminary notation. If $u \in C^{n}$, we can represent $u$ as a $2 n$-tuple $u=\left(x^{1}, y^{1}, x^{2}, y^{2}, \ldots, x^{n}, y^{n}\right)$ where each $x^{m}$ has the binary expansion $x^{m}=x_{1}^{m} x_{2}^{m} \cdots$ and $x_{k}^{m}=0$ if $k$ is even and the $y^{m}$ have similar expansions with $y_{k}^{m}=0$ for $k$ odd. Then we 
can define a mapping $\psi: C^{n} \rightarrow V^{n}$ by $\psi(u)=(x(\vec{i}), \vec{i})$, where

$$
i_{k}=\sum_{m=1}^{n} \frac{x_{k}^{m}+y_{k}^{m}}{2^{m-1}} \text {. }
$$

Theorem 3.1. The map $\psi: C^{n} \rightarrow V^{n}$ is a Lipschitz equivalence.

Proof. It follows from the uniqueness of representation of integers and the special properties of the points in $C^{n}$ that $\psi$ is one-to-one and onto. Let $u$ be as above, let $v=\left(w^{1}, z^{1}, w^{2}, z^{2}, \ldots, w^{n}, z^{n}\right)$ be a second point, and let $\psi(u)=(a, \vec{i}), \psi(v)=(b, \vec{j})$. By Proposition 2.1 the $m$ th coordinate of $a$ is $x^{m}+y^{m}$ and the $m$ th coordinate of $b$ is $w^{m}+z^{m}$, so we have

$$
\begin{aligned}
\|a-b\| & =\sum_{m=1}^{n}\left|x^{m}+y^{m}-w^{m}-z^{m}\right| \\
& \leq \sum_{m=1}^{n}\left|x^{m}-w^{m}\right|+\left|y^{m}-z^{m}\right|=\|u-v\| .
\end{aligned}
$$

Suppose that $k+1$ is the first index in which $\vec{i}$ and $\vec{j}$ disagree. Since $a$ and $b$ must have some coordinates that disagree in the $k+1$ st position of their binary expansions, $\|a-b\| \geq 1 / 2^{k+1}$. Therefore,

$$
\rho(\psi(u), \psi(v))=\|a-b\|+\frac{1}{2^{k}} \leq 3\|a-b\| \leq 3\|u-v\| .
$$

On the other hand,

$$
\|u-v\|=\sum_{m=1}^{n}\left|x^{m}-w^{m}\right|+\left|y^{m}-z^{m}\right| \leq \frac{2 n}{2^{k}},
$$

since $x^{m}, w^{m}$ and $y^{m}, z^{m}$ agree in at least their first $k$ places. Therefore

$$
\|u-v\| \leq 2 n\|a-b\|+\frac{2 n}{2^{k}}=2 n \rho(\psi(u), \psi(v)) .
$$

Corollary 3.1. If $X$ is a compact set in $\mathbf{R}^{n}$, there is a Lipschitz map of $C^{n}$ onto $X$.

\section{CONSTRUCTING IFSs}

Recall the contractions $h_{0}$ and $h_{1}$ on $\mathbf{R}^{2}$ constructed in $\S 3$. For an $n>1$ we index the members of the IFS $F^{n}$ as follows. For an integer $i \in \Gamma$, let $i=\sum_{k=0}^{n-1} x_{k} 2^{k}$ be its binary representation, and let $f_{i}=h_{x_{0}} \times h_{x_{1}} \times \cdots \times h_{x_{n-1}}$. For $i_{1}, i_{2}, \ldots, i_{k} \in \Gamma$, let $f_{i_{1} i_{2} \cdots i_{k}}$ be the composition $f_{i_{k}} f_{i_{k-1}} \cdots f_{i_{1}}$. It is easy to see that 
Proposition 4.1. Each $f_{i_{1} i_{2} \cdots i_{k}}$ is a contraction of $\mathbf{R}^{2 n}$ with contractivity constant $1 / 2^{k}$ and

$$
C^{n}=\bigcup_{i_{1}, i_{2}, \ldots, i_{k} \in \Gamma} f_{i_{1} i_{2} \cdots i_{k}}\left(C^{n}\right)
$$

for each $k$.

Given a compact set $X \subset \mathbf{R}^{n}$ with $0 \notin X$, let $C+X$ denote the union

$$
C+X=\left(C^{n} \times\{0\}\right) \cup(\{0\} \times X) \subset \mathbf{R}^{3 n} .
$$

Theorem 4.1. There is an IFS on $\mathbf{R}^{3 n}$ whose attractor is $C+X$.

Proof. Write $\mathbf{R}^{3 n}$ as $\mathbf{R}^{2 n} \times \mathbf{R}^{n}$ and let $\lambda: C^{n} \rightarrow X$ be a Lipschitz map with Lipschitz constant $L$. By the "Lipschitz Tietze Theorem" [5], we may assume that $\lambda$ is defined on all of $\mathbf{R}^{2 n}$. For each $i \in \Gamma$ let $\hat{f}_{i}$ be defined by

$$
\hat{f}_{i}(x, y)=\left(f_{i}(x), 0\right) \text {. }
$$

Choose a $k$ such that $L / 2^{k}<1$ and for each $k$-tuple $i_{1}, i_{2}, \ldots, i_{k} \in \Gamma$, let

$$
g_{i_{1} i_{2} \cdots i_{k}}(x, y)=\left(0, \lambda f_{i_{1} i_{2} \cdots i_{k}}(x)\right) \text {. }
$$

Then the collection

$$
\left\{\hat{f}_{i} \mid i \in \Gamma\right\} \cup\left\{\lambda g_{i_{1} i_{2} \ldots i_{k}} \mid i_{1}, i_{2}, \cdots, i_{k}\right\}
$$

is an IFS whose attractor is $C+X$.

\section{REFERENCES}

1. M. Barnsley, Fractals everywhere, Academic Press, Boston, MA, 1988.

2. K. Falconer, Fractal geometry, Wiley, New York, 1990.

3. M. Hata, On the structure of self-similar sets, Japan J. Appl. Math. 2 (1985), 381-414.

4. J. E. Hutchinson, Fractals and self similarity, Indiana Univ. Math. J. 30 (1981), 713-747.

5. J. Luukkainen and J. Väisälä, Elements of Lipschitz topology, Ann. Acad. Sci. Fenn. Ser. A 3 (1977), 85-122.

6. R. F. Williams, Composition of contractions, Bol. Soc. Brasil. Mat. 2 (1971), 55-59.

Department of Mathematics, University of North Carolina at Greensboro, GreensBORO, NORTH CAROLINA 27412

E-mail address: duvallp@hamlet.uncg.edu

Department of Mathematics, University of Tennessee, KNoxville, Tennessee 37996 E-mail address: husch@math.utk.edu 